"C 2019 IEEE. Personal use of this material is permitted. Permission from IEEE must be obtained for all other uses, in any current or future media, including reprinting/republishing this material for advertising or promotional purposes, creating new collective works, for resale or redistribution to servers or lists, or reuse of any copyrighted component of this work in other works." 


\title{
I-SEP: An Improved Routing Protocol for Heterogeneous WSN for IoT based Environmental Monitoring
}

\author{
Trupti Mayee Behera, Sushanta Kumar Mohapatra, Senior Member, IEEE, Umesh Chandra Samal, \\ Mohammad. S. Khan, Member, IEEE, Mahmoud Daneshmand, Life Member, IEEE, and \\ Amir H. Gandomi, Senior Member, IEEE
}

\begin{abstract}
Wireless Sensor Networks (WSN) is a virtual layer in the paradigm of the Internet of Things (IoT). It inter-relates information associated with the physical domain to the IoT drove computational systems. WSN provides ubiquitous access to location, the status of different entities of the environment, and data acquisition for long-term IoT monitoring. Since energy is a major constraint in the design process of a WSN, recent advances have led to project various energy-efficient protocols. Routing of data involves energy expenditure in considerable amount. In recent times, various heuristic clustering protocols have been discussed to solve the purpose. This article is an improvement of the existing Stable Election Protocol (SEP) that implements a threshold-based cluster head selection for a heterogeneous network. The threshold maintains uniform energy distribution between member and cluster head nodes. The sensor nodes are also categorized into three different types called normal, intermediate and advanced depending on the initial energy supply to distribute the network load evenly. The simulation result shows that the proposed scheme outperforms SEP and DEEC protocols with an improvement of $300 \%$ in network lifetime and $\mathbf{5 6 \%}$ in throughput.
\end{abstract}

Index Terms - WSN, Heterogeneous Network, CH selection, Network lifetime.

\section{INTRODUCTION}

$\mathrm{O}$ VER the last few years, the IoT paradigm has evolved as one of the biggest technological advances of modern science. With the evolving era of WiFi and 4G-LTE wireless access of Internet [1] [2], IoT enabled devices like computer, tablets, mobile phones are able to access information about the environment and other objects without human intervention. The two key enablers of IoT are Radio frequency identification (RFID) and WSN.

A WSN constitutes spatially dispersed sensor nodes meant

Manuscript received XXX XX, XXXX; accepted XXX XX, XXXX. Date of publication XXXX XX, XXXX; date of current version XXXX XX, XXXX.

T. M. Behera, S. K. Mohapatra, and U. C. Samal are with School of Electronics Engg., KIIT University, Bhubaneswar, 751024, India. (e-mail: truptifet@kiit.ac.in ; skmctc74@ gmail.com; umesh.samalfet@kiit.ac.in ).

M. S. Khan is with Department of Computer \& Information Sciences, East Tennessee State University, Johnson City, USA (e-mail: adhoc.khan@gmail.com ).

M. Daneshmand is with the School of Business, Stevens Institute of Technology, Hoboken, NJ 07030, USA and he is Steering Committee Chair of IEEE IoT J (e-mail: Mahmoud.Daneshmand@ stevens.edu )

A.H. Gandomi (corresponding author) is with the Faculty of Engineering and Information Technology, University of Technology Sydney, Sydney, NSW 2007, Australia (e-mail: gandomi@uts.edu.au).

Digital Object Identifier 10.1109/JIOT.2019.2940988 to record and monitor various physical and environmental conditions with low-cost data acquisition. The sensor nodes are usually energy deprived in nature which leads to the formulation of innovative techniques to limit any unnecessary energy dissipation mounting to shortening of lifetime [3]. The source nodes consume a lot of energy in communicating data directly to BS. Hence they have to depend on intermediate nodes to operate. A comparative study of routing protocols [4][5][6] indicates different methods to enhance network lifetime. Khalil et al. [7] have designed a dynamic clustering scheme to reduce energy consumption while transferring information thereby maintaining a trade-off between stability period and lifetime of the network. The method assumes only single-hop data transfer which may not be feasible for a large scale network in an IoT system. Dynamic clustering used in [8] is based on multi-hop communication, where sensors transfer data via intermediate nodes to sink, but reducing the energy at node level is still a problem.

Clustering algorithms have emerged as the most energyefficient communication protocol that groups the sensor nodes in clusters. Each cluster is headed by a cluster head $(\mathrm{CH})$ responsible for data collection from sensing nodes. The $\mathrm{CH}$ fuses the data to remove any redundancy and then transfers to the sink node or BS. Hence election of $\mathrm{CH}$ should be done judiciously to maintain proper network balance for energy management. The existing routing methods that select $\mathrm{CHs}$ optimally may not be suitable for large scale environments where WSN is integrated with IoT.

Any ad-hoc system can be either heterogeneous or homogeneous. The network where sensor nodes are supplied with equal amount of energy termed as homogeneous, is shown in Fig. 1(a). Heterogeneous networks as shown in Fig. 1(b) and (c) have uneven initial energy distribution. A group of nodes called advanced nodes has higher energy in comparison to normal nodes. The proposed network model introduces intermediate nodes, along with normal and intermediate nodes, that have energy in between normal and advanced nodes. When all sensors in the network start with a constant energy level, the nodes die out randomly within a short span of time. Heterogeneous network structures are gaining importance because it delivers better network performance without demanding much increase in cost [9].

One of the challenging IoT application domains is environmental monitoring, where the sensors are deployed in 
harsh operating conditions [10] [11]. Fig. 2 depicts a scenario where sensor nodes are placed over soil, air, and water to deliver feasible or even optimal solution to monitor moisture, humidity, $\mathrm{pH}$ level, temperature.

(a)

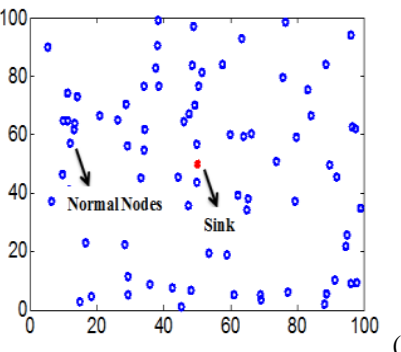

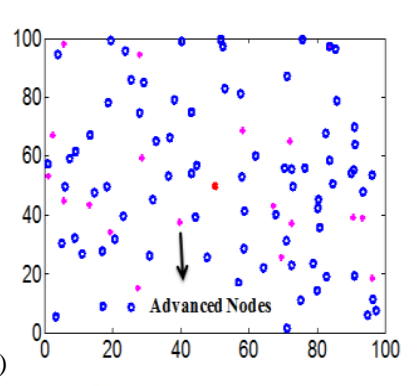

(b) (c)

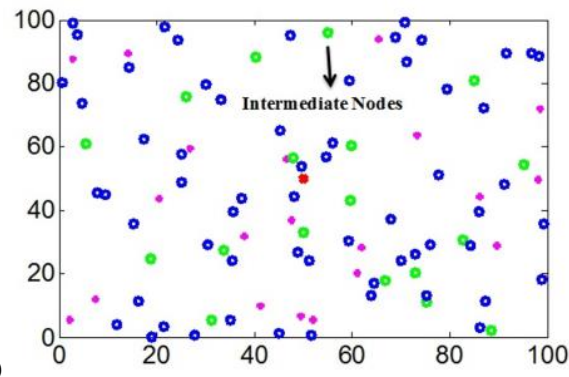

Fig. 1. (a) Homogenous (b) Heterogeneous (c) Proposed Network Model

After deployment, the sensors are expected to keep sensing the environment for a longer period and have no scope of recharging the node battery. Furthermore, the cost and difficulty of accessing the field physically for deployment and maintenance [1] become a challenging task. The WSN platform should offer low-cost nodes with long unattended service time and minimal maintenance to overcome these issues. This is possible only if the network comprises sensors with two or more initial energy levels, which is one of the important criteria considered in the proposed method. Hence it can be inferred that heterogeneous networks perform better when applied in the IoT environment.

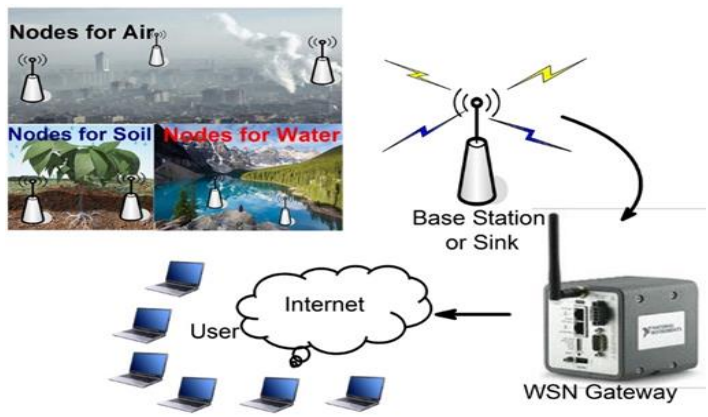

Fig. 2. Environmental Monitoring using WSN

LEACH (Low Energy Adaptive Clustering Hierarchy) is a fully distributed routing algorithm based on TDMA designed for homogeneous networks [12]. SEP (Stable election protocol) is a variant of LEACH where certain populations of nodes (advanced nodes) have some additional energy than other nodes (normal) within the same network [9]. This article discusses an extension of SEP that intends to maximize the network lifetime and throughput by introducing a threshold level in the $\mathrm{CH}$ selection process that can be applied in a WSN based IoT network.
Starting with the introduction, a brief analysis of related work is presented in the next section. Section III enlightens the network model and the proposed scheme is provided in section IV. Section V discusses simulation results with issue related analysis. And finally, the conclusion is drawn.

\section{RELATED WORK}

Any clustering algorithm functions by segmenting the field into clusters headed by a $\mathrm{CH}$. The member nodes, ie, non- $\mathrm{CH}$ nodes communicate their data to $\mathrm{CH}$, where the data is processed and aggregated to remove redundancy and sent to the BS. As energy consumption is distributed evenly throughout the network, the overall energy consumption is said to be reduced [13].

LEACH is an energy constrained protocol [14]. The initial $\mathrm{CH}$ selection is done randomly such that every node has the chance of becoming $\mathrm{CH}$ once in every $1 / \mathrm{p}$ epoch [15]. In subsequent rounds, a random number is generated in the range $[0,1]$ and only if the number is less than threshold $T_{n}$, formulated by (1), the node functions as $\mathrm{CH}$ [16]. The next $\mathrm{CH}$ is chosen from the set of non-CH nodes $G$.

$$
T_{n}=\left\{\begin{array}{l}
\frac{p}{1-p(r \bmod 1 / p)} ; \text { for } \mathrm{n} \in \mathrm{G} \\
0 ; \text { Otherwise }
\end{array}\right.
$$

After the cluster formation stage, all the elected $\mathrm{CHs}$ broadcast a TDMA schedule for non-CH nodes. The sensing nodes transmit the data during the specific allotted time slots. Once the transmission process completes, the frame repeats. Although LEACH is a distributed protocol, it may not be beneficial for large-scale application due to energy constraint.

In a heterogeneous network [9] of $n$ nodes, $a$ fraction of nodes has the extra energy of factor $\alpha$ that are termed advanced nodes. The SEP algorithm focuses on the weighted election probabilities of each node for the election of $\mathrm{CHs}$ according to their respective energies. It ensures a more extended stability period with better performance than that of LEACH protocol. The DEEC protocol [17] was proposed for networks with different energy level, where the selection of $\mathrm{CH}$ can be decided by both initial as well as residual energy [18]. An enhanced SEP was proposed in [19] that deployed three categories of sensors based on energy levels; named normal, intermediate and advanced nodes. Due to the threetier node scenario, the network lifetime is enhanced; however, the quantity of $\mathrm{CHs}$ in each cluster could not be controlled.

EEHC is an energy-efficient heterogeneous clustering scheme that elects $\mathrm{CHs}$ considering the weights of each sensor [20]. The residual energy of each node decides the set of probable $\mathrm{CHs}$ in the heterogeneous network. MATLAB results indicate enhanced network lifetime in comparison to LEACH. However, no result analysis is done with any heterogeneous algorithms. Threshold sensitive SEP (T-SEP) is a reactive protocol introduced in [21], where data is transmitted by sensors only when the explicit threshold is reached. Three level heterogeneous nodes were deployed to study the lifetime and stability period of the network. Another modification of SEP for fog-supported WSN discussed in [22] maintains a 
balanced energy dissipation to prolong the network lifetime. Both types of nodes have equal probabilities to be elected as $\mathrm{CHs}$. As the $\mathrm{CHs}$ are chosen judiciously, the node death rate decreases in comparison to LEACH-DCHS [12] and other modifications of SEP.

I-LEACH was proposed in [23] to introduce threshold based $\mathrm{CH}$ selection, where the LEACH protocol was modified to obtain better results for IoT based applications. The simulation result showed better performance for different scenarios in comparison to energy efficient routing protocols like LEACH, EECS [24], CPCHSA [25] and Mod-LEACH [26]. However, the algorithm was designed only for homogeneous networks and cannot be implemented for heterogeneous scenarios. To overcome high system complexity CREEP scheme was proposed in [27] that selects numerous $\mathrm{CHs}$ to improve the network lifetime by modifying threshold value in a 2-level heterogeneous WSN. Unbalanced energy consumption near $\mathrm{CHs}$ limit the network lifetime; particle swarm optimization based $\mathrm{CH}$ selection was proposed in [28] that enhances lifetime by identifying energy holes. The approach assumes homogenous network where nodes die out randomly.

In SEP routing algorithm, the election of new $\mathrm{CH}$ with the formation of new clusters is done regularly for each round. This in return leads to unnecessary energy utilization generated due to routing overhead which will affect the performance of IoT devices [29] connected to the sensor network. According to the classical SEP algorithm, a $\mathrm{CH}$ in the current round will not be able to participate in the $\mathrm{CH}$ election process in the next round [27]. However, there can be cases where a $\mathrm{CH}$ has not utilized an ample amount of energy in the preliminary round and is eligible for the $\mathrm{CH}$ election process in the next round. It can also happen that a sensor with a comparatively lesser amount of energy gets elected as $\mathrm{CH}$ in the subsequent selection process [26] that leads to the untimely death of the network. Also, new $\mathrm{CH}$ requires new cluster formation in each round, which consumes the node power in sending messages like ADV (advertisement) and ACK (acknowledgment) to CHs back and forth. The above limitation in SEP motivates to investigate and establish an efficient $\mathrm{CH}$ replacement method.

The key contributions of the proposed work are:

i. The article aims to enhance the fundamental SEP algorithm by incorporating a unique threshold strategy for $\mathrm{CH}$ selection.

ii. The proposed method aims to reduce extra power consumption by avoiding unnecessary clusters and $\mathrm{CHs}$ formation in each round.

iii. After $\mathrm{CH}$ selection, the proposed algorithm assigns a high level of energy to the node. For the subsequent rounds, when the node again becomes a sensing node, the low level of energy will be assigned. This variation of energy level for different nodes will be beneficial for maintaining proper energy distribution in the network.

\section{SYSTEM MODEL}

Considering energy efficiency and energy balancing [28] as the most vital parameter in the design process of any routing algorithm in WSN, an advanced technique to route data in a heterogeneous network is introduced. To control the energy dissipation, three-level heterogeneity with respect to initial node energy is considered. All the nodes are static in nature. Advance nodes have the maximum, and normal nodes have the lowest level of energy. Intermediate nodes are the ones with higher energy than normal nodes and lower than advanced nodes. Let $b$ be the section of nodes that are assigned an intermediate energy level with $\beta$ times more power than normal ones, where $\beta=\alpha / 2$.

$E_{0}$ represents the initial energy given to normal nodes. The advanced and intermediate nodes have $E_{0}(1+\alpha)$ and $E_{0}(1+\beta)$ energies respectively. Hence, the total energy of each type of node can be summed up as:

$$
\begin{aligned}
E_{N} & =n E_{0}(1-a-b) \\
E_{I} & =n b E_{0}(1+\beta) \\
E_{A} & =n a E_{0}(1+\alpha)
\end{aligned}
$$

Where, $E_{N}, E_{I}$, and $E_{A}$ are the energies for normal, intermediate and advanced nodes respectively. Therefore, the overall energy of the three types of nodes written as

$$
\begin{aligned}
E_{\text {Total }} & =n E_{0}(1-a-b)+n b E_{0}(1+\beta)+n a E_{0}(1+\alpha) \\
& =n E_{0}(1+a \alpha+b \beta)
\end{aligned}
$$

The $\mathrm{CH}$ election process is similar to that of LEACH and SEP. The threshold value for $\mathrm{CH}$ selection is formulated for each type of node by considering their probabilities. Let $G_{1}, G_{2}$ and $G_{3}$ represent the set of nodes in each category that had not performed as $\mathrm{CH}$ in former epochs and $r$ represents the current round. Considering $p_{(N)}, p_{(I)}$ and $p_{(A)}$ as the probabilities of normal, intermediate and advanced nodes to be elected as $\mathrm{CHs}$ respectively. For normal nodes,

$$
\begin{gathered}
p_{(\mathrm{N})}=\frac{p}{1+a \alpha+b \beta} \\
T_{\left(\mathrm{n}_{N}\right)}=\left\{\begin{array}{l}
\frac{p_{(N)}}{1-p_{(N)}\left(r \text { mod } 1 / p_{(N)}\right)} ; \text { if } \mathrm{n}_{N} \in G_{1} \\
0 ; \text { Otherwise }
\end{array}\right.
\end{gathered}
$$

For intermediate nodes,

$$
\begin{gathered}
p_{(\mathrm{I})}=\frac{\mathrm{p}(1+\beta)}{1+a \alpha+b \beta} \\
T_{\left(\mathrm{n}_{I}\right)}=\left\{\begin{array}{l}
\frac{p_{(\mathrm{I})}}{1-p_{(\mathrm{I})}\left(r \bmod 1 / p_{(\mathrm{I})}\right)} \\
0 ; \text { Otherwise }
\end{array}\right.
\end{gathered}
$$

For advance nodes, $p_{(\mathrm{A})}=\frac{\mathrm{p}(1+\alpha)}{1+a \alpha+b \beta}$

$$
T_{\left(\mathrm{n}_{A}\right)}=\left\{\begin{array}{l}
\frac{p_{(\mathrm{A})}}{1-p_{(\mathrm{A})}\left(r \text { mod } 1 / p_{(\mathrm{A})}\right)} ; \text { if } \mathrm{n}_{A} \in G_{3} \\
0 ; \text { Otherwise }
\end{array}\right.
$$

Now, from equation (6), (8) and (10), we can find the average total $\mathrm{CHs}$ per round as:

$$
n(1-a-b) p_{(N)}+n a p_{(\mathrm{A})}+n b_{(I)}=n p
$$

It can be inferred from (12) that the resultant of $\mathrm{CHs}$ in a heterogeneous environment is equal to that in case of LEACH protocol. However, the energy dissipation is controlled in a better way owing to the heterogeneous energy level [21]. 


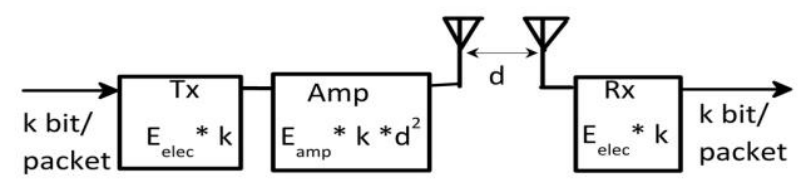

Fig. 3. The first-order radio communication system

The communication in the heterogeneous network follows the model shown in Fig. 3 [30]. If $d$ (Euclidean distance between sending and receiving nodes) is less than or equal to a reference distance $d_{0}$ (where $d_{0}=\sqrt{E_{f s} / E_{m p}}$ ) then energy dissipation is calculated using multi-path fading model otherwise free-space model is used. Assuming symmetrical communication channel where the energy expended by a sensing node in transmitting ' $k$ ' bits per packet can be given as in [31] [32]:

$$
\begin{aligned}
& E_{T x}(k, d)=E_{T x_{-} \text {elec }}(k)+E_{T x_{-} m p}(k, d) \\
& E_{T x}(k, d)=\left\{\begin{array}{l}
E_{\text {elec }} \times k+E_{f s} \times k \times \mathrm{d}^{2}, d \leq d_{0} \\
E_{\text {elec }} \times k+E_{m p} \times k \times \mathrm{d}^{4}, d>d_{0}
\end{array}\right.
\end{aligned}
$$

$E_{m p}$ and $E_{f s}$ are the amplifier parameters of transmission for multi-path fading model and free-space model respectively [33]. If the transmitter or receiver expends $E_{\text {elec }}$ amount of energy per bit, then to receive a packet of $k$ bits, a sensor node expends $E_{R x}(k)$ energy given as:

$$
E_{R x}(k)=E_{R x_{-} \text {elec }}(k)+k E_{\text {elec }}
$$

The energy dissipation is estimated in each round for the calculation of threshold boundary for $\mathrm{CH}$ election. The proposed scheme aims to estimate a threshold energy value to be maintained by all types of nodes to preserve energy for the network longevity.

\section{PROPOSED WORK}

Once the $\mathrm{CH}$ is selected, the sensing nodes join the $\mathrm{CH}$ according to the information in the ADV message sent by them. Hence for each round, the $\mathrm{CHs}$ and clusters keep changing. In case, some $\mathrm{CHs}$ (either normal, intermediate or advanced) especially those near to the BS, need not use much of its energy in transmitting data packets. These $\mathrm{CHs}$ can continue to transmit with the same group of member nodes in the next round also. But according to conventional SEP, these nodes will not be able to partake in the $\mathrm{CH}$ election process for the next $1 / p$ epochs.

The proposed work introduces a threshold energy value for each type of node in the SEP algorithm, now termed as I-SEP (IoT-SEP). In I-SEP, the threshold energy value decides whether the $\mathrm{CH}$ and the corresponding cluster should change or continue transmitting in the next round. After each round, the $\mathrm{CH}$ node residual energy is evaluated. If the residual energy is less than the estimated threshold, the $\mathrm{CH}$ election process initiates and new clusters are formed. This controls the energy wasted in the unnecessary transfer of routing information for new $\mathrm{CH}$ and also reduces the extra energy consumed in new cluster formation.

The energy requirement of a sensing node and a $\mathrm{CH}$ node will never be the same. $\mathrm{CHs}$ perform extra functions like aggregation and fusion, hence it is desirable that they should be equipped with higher energy level in comparison to those nodes meant for intra-cluster communication. This will additionally save power and packet drop ratio. Hence, the modified algorithm assigns a high energy amplification level only for the selected $\mathrm{CH}$. In the next round, if the $\mathrm{CH}$ switches to a normal sensing node, the modified algorithm assigns a low energy level [31][23] to the corresponding node.

With $n$ number of total nodes in the network and $C$ be the percentage of clusters and $R$ is the $\mathrm{CH}$ replacement count. $P_{k T x}$ and $P_{k R x}$ are the packet size at transmission and reception.

Let $N=n C$ represents the size of each cluster.

The $\mathrm{CH}$ replacement process for new cluster formation also utilizes some energy given as $P_{H R}$, such that

$$
P_{H R}=\left\{P_{k T x} P_{T x}+P_{k R x} P_{R x}(n C-1)\right\} \times R N
$$

Where $P_{T x}=$ Energy spent in transmitting 1 Byte of data and $P_{R x}=$ Energy spent in the receiving 1 Byte of data.

The power utilization of each cluster $P_{W E C}$ can be estimated by the multiplying the initial energy supplied to each category of a node with the cluster size, that is,

$$
\begin{aligned}
& P_{W E C(N)}=E_{0} \times n C \\
& P_{W E C(\mathrm{I})}=E_{0}(1+\beta) \times n C \\
& P_{W E C(\mathrm{~A})}=E_{0}(1+\alpha) \times n C
\end{aligned}
$$

The power consumption in each cluster $i$ for a round can be found by estimating the energy cost of a node in both cases, i.e. when it acts as a sensing node and as a $\mathrm{CH}$. Consequently,

$$
\begin{aligned}
P_{H R}(i)= & \left\{\left(N_{i}-1\right) P_{k T x} P_{T x} \times P_{k R x} P_{R x}\right\}+ \\
& \left\{\left(N_{i}-1\right) P_{k R x} P_{R x}+\left(N_{i}-1\right) P_{k T x} P_{T x}\right\} \\
= & n\left(5 N_{i}-3\right) P_{T x}
\end{aligned}
$$

The sensing node expands the $n P_{T x}$ amount of energy during transmission to the respective CHs. When not transmitting, the nodes move to sleep mode by switching the radio off till the next TDMA slot. The CHs consumes $n(N-1) P_{R x}$ energy in the process of data fusion and aggregation. The $\mathrm{CH}$ then transmits the fused data to the $\mathrm{BS}$ expending $n(N-1) P_{T x}$ energy. To estimate the threshold value for $\mathrm{CH}$ replacement, the information regarding the number of rounds must be known. Count $_{\text {Rnd }}$ represents the total number of iterations in the network which can be calculated for the three types of nodes.

$$
\begin{aligned}
& \text { Count }_{R n d(N)}=\frac{P_{H R}}{P_{W E C(N)}} \times 100 \\
& \text { Count }_{R n d(\mathrm{I})}=\frac{P_{H R}}{P_{W E C(\mathrm{I})}} \times 100 \\
& \text { Count }_{R n d(\mathrm{~A})}=\frac{P_{H R}}{P_{W E C(\mathrm{~A})}} \times 100
\end{aligned}
$$

From equation (21) and (22-24), the threshold power level can be calculated as:

$$
\begin{gathered}
P_{T h(N)}=\text { Count }_{R n d(N)}\left(P_{k T x}+P_{k R x}\right) P_{T x} \\
P_{T h(\mathrm{I})}=\text { Count }_{R n d(\mathrm{I})}\left(P_{k T x}+P_{k R x}\right) P_{T x} \\
P_{T h(\mathrm{~A})}=\text { Count }_{R n d(\mathrm{~A})}\left(P_{k T x}+P_{k R x}\right) P_{T x}
\end{gathered}
$$

$P_{T h(N)}, P_{T h(I)}$ and $P_{T h(A)}$ are the threshold values for normal, intermediate and advanced node respectively. The introduction of a threshold value of $\mathrm{CH}$ replacement for each type of node in the modified SEP algorithm improves WSN lifetime by 
minimizing the total network energy. The algorithm for the proposed algorithm I-SEP is included in Table I.

TABLE I

I-SEP ALGORITHM

\begin{tabular}{l}
\hline \hline $\mathrm{P}_{\mathrm{Th}}:$ Threshold for $\mathrm{CH}$ election \\
$\mathrm{E}_{\mathrm{Res}}:$ Residual energy of existing $\mathrm{CH}$ \\
$\mathrm{CH}: \mathrm{CH}$ counter \\
$\mathrm{AF}:$ Amplification factor \\
$G_{i}:$ Set of non-CH nodes
\end{tabular}

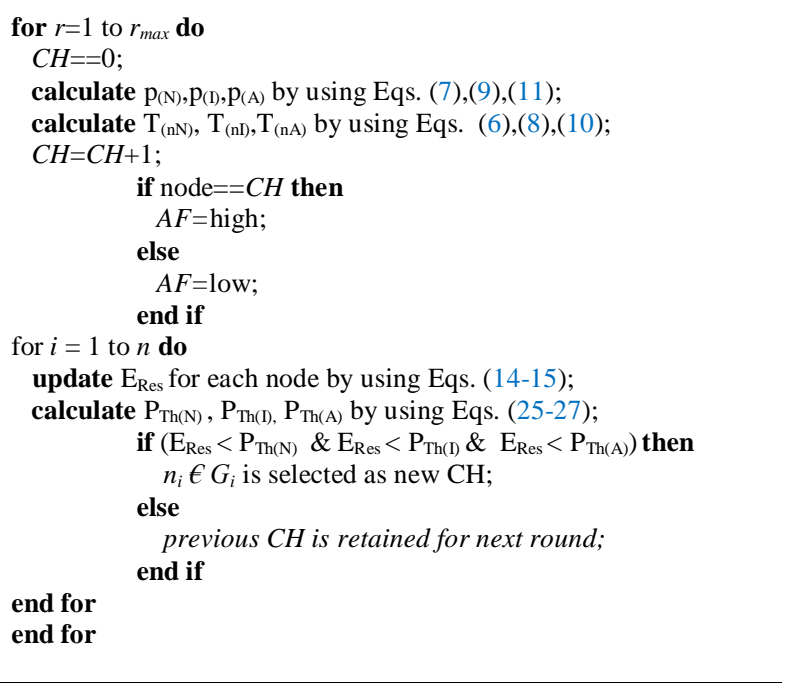

\section{Simulation Result \& Discussions}

Simulations were carried out in MATLAB with 100 sensor nodes deployed in a network of $100 \times 100 \mathrm{~m}^{2}$. The BS is positioned at the center with unlimited energy. The network parameters used for simulation are enlisted in Table II. To analyze the behavior of the proposed model in comparison to SEP [9] and DEEC [17] that follows two level heterogeneity, the values of ' $a$ ' and ' $\alpha$ ' are varied while ' $b$ ' maintains a constant value of 0.3 . For the first instance $\alpha=1, a=0.1$, for second case $\alpha=2$, and $\mathrm{a}=0.1$, for third case $\alpha=1, \mathrm{a}=0.2$ and last case $\alpha=2, \mathrm{a}=0.2$.

TABLE II

\section{SIMULATION PARAMETERS}

\begin{tabular}{ll}
\hline \hline Parameters & Value \\
\hline $\mathrm{P}_{\mathrm{Tx}}, \mathrm{P}_{\mathrm{Rx}}$ (The total energy of the network) & $50 \mathrm{~J}$ \\
$\mathrm{E}_{\mathrm{mp}}$ (Energy dissipation: receiving) & $0.0013 \mathrm{pJ} / \mathrm{bit} / \mathrm{m}^{4}$ \\
$\mathrm{E}_{\mathrm{fs}}$ (Energy dissipation: free space model) & $10 \mathrm{pJ} / \mathrm{bit} / \mathrm{m}^{2}$ \\
$\mathrm{E}_{\text {amp }}$ (Energy dissipation: power amplifier) & $100 \mathrm{pJ} / \mathrm{bit} / \mathrm{m}^{2}$ \\
$\mathrm{E}_{\mathrm{DA}}$ (Energy dissipation: aggregation) & $5 \mathrm{~nJ} / \mathrm{bit}$ \\
$\mathrm{d}_{0}$ (Reference distance) & $87 \mathrm{~meters}$ \\
$\mathrm{k}$ (Packet size) & $4000 \mathrm{bits}$ \\
\hline \hline
\end{tabular}

The efficiency of any routing algorithm can be analyzed by estimating the number of data packets communicated to the sink node or BS with minimum packet drop ratio. This is called network throughput. With $10 \%$ advanced nodes in the network, the throughput and network lifetime are analyzed for SEP and DEEC as shown in Fig. 4 and 5 respectively. It can be found the throughput, increases considerably for I-SEP as compared to both protocols. This improvement results due to the limitation in data transmission for the proposed I-SEP.
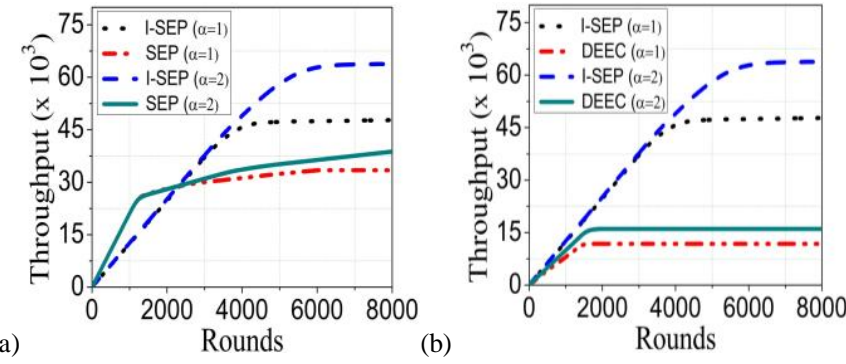

Fig. 4. Throughput for $\mathbf{a}=\mathbf{0 . 1}$ in comparison to (a) $\operatorname{SEP}$ (b) DEEC

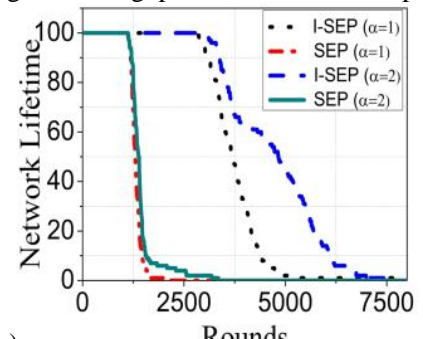

(a)

Rounds

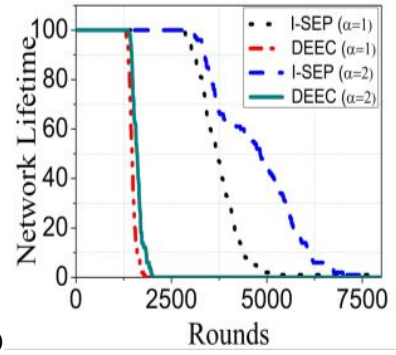

(b)

Fig. 5. Network Lifetime for $\mathbf{a}=\mathbf{0 . 1}$ in comparison to (a) SEP (b) DEEC

The efficient threshold based $\mathrm{CH}$ replacement saves energy owing to the dual power level assignment for $\mathrm{CH}$ node and sensing nodes. Introducing a threshold to retain the $\mathrm{CH}$ with high residual energy helps to conserve energy for each category of nodes. The nodes are thereby able to communicate more data over a longer period of time. When $\alpha$ is kept constant at 1 , the throughput of I-SEP increased by $50 \%$ and $240 \%$ as that of SEP and DEEC respectively. Similarly for $\alpha$ changed to 2 , the maximum throughput further increases by $56 \%$ and $300 \%$ in comparison to SEP and DEEC respectively.
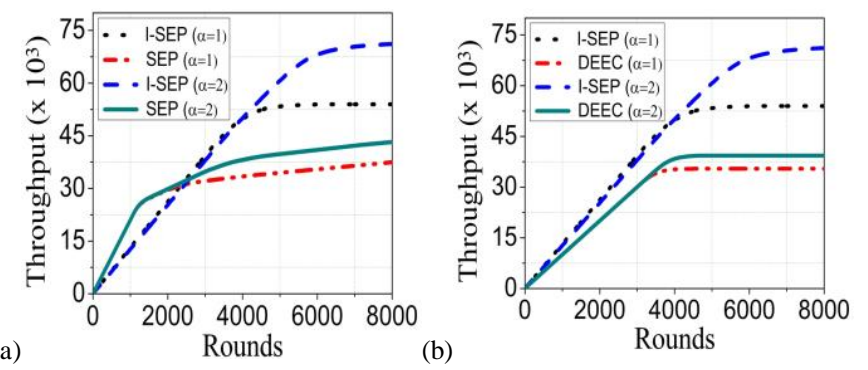

Fig. 6. Throughput for $\mathbf{a}=\mathbf{0 . 2}$ in comparison to (a) SEP (b) DEEC

Fig. 6 shows the throughput achieved for SEP, I-SEP, and DEEC for the case when the percentage of advanced nodes is increased to $20 \%$. With more number of advanced nodes, the number of $\mathrm{CHs}$ from this category of nodes also increases which indicate more data transfer. Hence, the maximum packets send to BS for I-SEP increases considerably by $53 \%$ and 67\% than SEP for $\alpha$ value of 1 and 2 respectively. On a similar manner, when compared to DEEC, the rise is $32 \%$ and $80 \%$ for $\alpha=1$ and $\alpha=2$ respectively.

The instant node starts sensing in the network until the death of the last node is termed as stability period [17]. In subsequent rounds, the sensor nodes deplete energy and die eventually. Owing to the availability of sensors with additional levels of energy, I-SEP performs better in context of lifetime than SEP. Since the transmission rate is less in I-SEP, so energy consumption will be less resulting in extended network lifetime. 


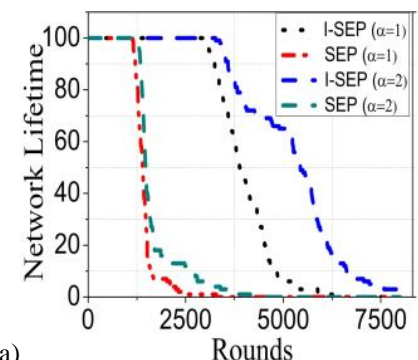

(a) Rounds

(b)

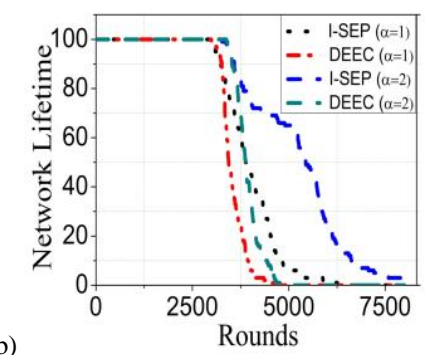

Fig. 7. Network Lifetime for $\mathbf{a}=\mathbf{0 . 2}$ in comparison to (a) SEP (b) DEEC

Also, I-SEP considers three categories of nodes whereas SEP and DEEC have only two types of nodes based on initial energy. This heterogeneity in energy level contributes to extend the network longevity to more number of rounds as shown in Fig. 5 and 7. The lifetime metrics in terms of Last Node Dead (LND) and First Node Dead (FND) for different values of $\alpha$ and $a$ is shown in Table III.

TABLE III

LIFETIME METRICS

\begin{tabular}{|c|c|c|c|c|}
\hline $\mathbf{a}$ & Protocol & $\alpha$ & FND & LND \\
\hline \multirow{6}{*}{0.1} & \multirow{2}{*}{ SEP } & 1 & 1028 & 2023 \\
\hline & & 2 & 1125 & 3348 \\
\hline & \multirow{2}{*}{ DEEC } & 1 & 1308 & 1809 \\
\hline & & 2 & 1371 & 2002 \\
\hline & \multirow{2}{*}{ I-SEP } & 1 & 2846 & 5287 \\
\hline & & 2 & 2876 & 7600 \\
\hline \multirow{6}{*}{0.2} & \multirow{2}{*}{ SEP } & 1 & 1141 & 3289 \\
\hline & & 2 & 1259 & 4161 \\
\hline & \multirow{2}{*}{ DEEC } & 1 & 2997 & 4579 \\
\hline & & 2 & 3388 & 4938 \\
\hline & \multirow{2}{*}{ I-SEP } & 1 & 2997 & 6258 \\
\hline & & 2 & 3268 & $<8000$ \\
\hline
\end{tabular}

Since the energy requirement for $\mathrm{CH}$ and sensing nodes are not the same, I-SEP assigns different power levels for these categories of nodes. From the table data, it is clear that by increasing the advanced nodes count, the stability period and network lifetime can be increased, owing to the segregation of nodes into different power levels. The average number of nodes dead in each category is shown in Fig. 8.

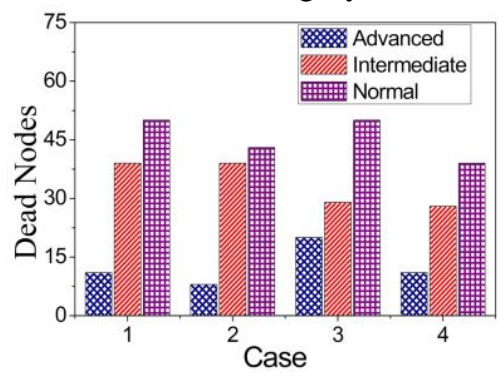

Fig. 8. Dead Nodes in I-SEP

Since the highest energy level is supplied to advanced nodes, the number of dead nodes is less in this category as compared to intermediate and normal nodes. With time, the normal nodes tend to die out at a faster rate. Henceforth, the intermediate and advanced nodes get elected as $\mathrm{CH}$ that stretches the lifetime to more number of rounds and increases the $\mathrm{CH}$ count as shown in Fig. 9.
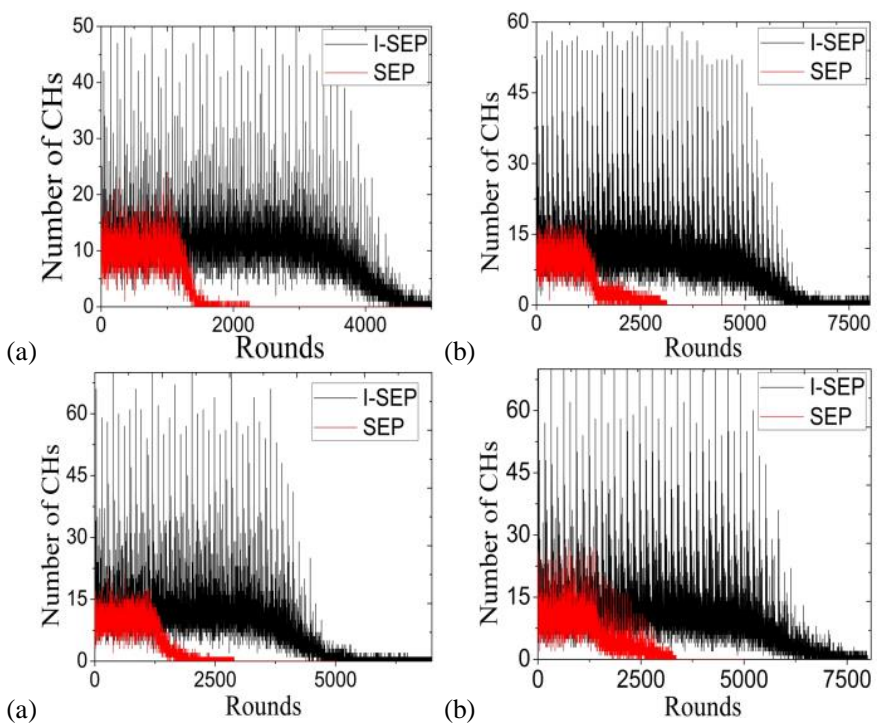

Fig. 9. CH count (a) Case 1 (b) Case 2 (c) Case 3 (d) Case 4

$\mathrm{CHs}$ are responsible for aggregating and routing of data to the BS. Fig. 9 shows the $\mathrm{CH}$ formation in each round. In all the cases, the $\mathrm{CH}$ count is much higher for I-SEP than SEP which indicates more data transfer to BS resulting in increased throughput. When $10 \%$ of total nodes are advanced nodes, the $\mathrm{CH}$ count for I-SEP reaches almost 50 and 60 in each round for $\alpha$ value of 1 and 2 respectively as compared to only 20 for SEP. The strength starts decreasing beyond 5000 rounds for $\alpha=1$, and 6000 rounds for $\alpha=2$ which indicates an enhancement in network lifespan. Similarly, for a network with $20 \%$ advanced nodes, $\mathrm{CH}$ count reaches 70 to 80 for I$\mathrm{SEP}$ as compared to 20 to 25 for SEP. The $\mathrm{CH}$ strength decreases beyond 6000 rounds for $\alpha=1$, and 7000 rounds for $\alpha=2$. The increased $\mathrm{CH}$ count is a result of heterogeneity. Since the network has nodes of three different level of energy and $\mathrm{CHs}$ are chosen from each type of node for equal energy distribution, the overall network energy depletes at a slower rate. As a result, $\mathrm{CHs}$ continue sending data packets to $\mathrm{BS}$ for more rounds, thereby enhancing network performances.

\section{CONCLUSIONS AND FUTURE SCOPE}

Stability period, network lifetime and throughput are the key factors in the design of a routing protocol for WSN. To evaluate the proposed modified algorithm, extensive simulations have been conducted to confirm the advantages of implementing the protocol practically. A distributed routing algorithm is illustrated that will be well suited for a heterogeneous network where sensors are deployed with more than one energy level. The proposed algorithm is a modification of SEP, and well suited for IoT based environmental monitoring. The simulation result shows I-SEP outperforms protocols like SEP and DEEC in terms of lifetime and throughput for various values of node density. The protocol also switches energy levels between $\mathrm{CH}$ nodes and member nodes, which also contribute to saving energy of the network. Implementing the modified algorithm for a mobile network where nodes move from one point to another with a constant speed can be explored in the future. 


\section{REFERENCES}

[1] M. T. Lazarescu, "Design of a WSN platform for long-term environmental monitoring for IoT applications," IEEE J. Emerg. Sel. Top. Circuits Syst., vol. 3, no. 1, pp. 45-54, 2013.

[2] J. Gubbi, R. Buyya, S. Marusic, and M. Palaniswami, "Internet of Things (IoT): A vision, architectural elements, and future directions," Futur. Gener. Comput. Syst., vol. 29, no. 7, pp. 1645-1660, 2013.

[3] A. A. Bara'a and E. A. Khalil, "A new evolutionary based routing protocol for clustered heterogeneous wireless sensor networks," Appl. Soft Comput., vol. 12, no. 7, pp. 1950-1957, 2012.

[4] S. K. Singh, P. Kumar, and J. P. Singh, "A Survey on Successors of LEACH Protocol," IEEE Access, vol. 5, pp. 4298-4328, 2017.

[5] D. Goyal and M. R. Tripathy, "Routing protocols in wireless sensor networks: a survey," in Advanced Computing \& Communication Technologies (ACCT), 2012 Second International Conference on, 2012, pp. 474-480.

[6] T. M. Behera, U. C. Samal, and S. K. Mohapatra, "Routing protocols," in Studies in Computational Intelligence, vol. 776, 2019.

[7] E. A. Khalil and A. A. Bara'a, "Energy-aware evolutionary routing protocol for dynamic clustering of wireless sensor networks," Swarm Evol. Comput., vol. 1, no. 4, pp. 195-203, 2011.

[8] S. Wang, J. Yu, M. Atiquzzaman, H. Chen, and L. Ni, "CRPD: a novel clustering routing protocol for dynamic wireless sensor networks," Pers. Ubiquitous Comput., vol. 22, no. 3, pp. 545-559, 2018.

[9] G. Smaragdakis, I. Matta, and A. Bestavros, "SEP: A stable election protocol for clustered heterogeneous wireless sensor networks," 2004.

[10] A. Whitmore, A. Agarwal, and L. Da Xu, "The Internet of Things-A survey of topics and trends," Inf. Syst. Front., vol. 17, no. 2, pp. 261274, 2015.

[11] D. M. Swati D., Rajasekhara M., Gandomi A.H., Patan R., "Internet of Things Mobile - Air Pollution Monitoring System (IoT-Mobair)," IEEE Internet Things J., vol. In press, 2019.

[12] M. J. Handy, M. Haase, and D. Timmermann, "Low energy adaptive clustering hierarchy with deterministic cluster-head selection," in Mobile and Wireless Communications Network, 2002. 4th International Workshop on, 2002, pp. 368-372.

[13] S. Selvakennedy, S. Sinnappan, and Y. Shang, "A biologically-inspired clustering protocol for wireless sensor networks," Comput. Commun., vol. 30, no. 14-15, pp. 2786-2801, 2007.

[14] T. Gao, L. Zhang, Y. Gai, and X. Shan, "Load-Balanced cluster-based cooperative MIMO transmission for wireless sensor networks," in Wireless Communication Systems, 2007. ISWCS 2007. 4th International Symposium on, 2007, pp. 602-606.

[15] W. B. Heinzelman, A. P. Chandrakasan, and H. Balakrishnan, "An application-specific protocol architecture for wireless microsensor networks," IEEE Trans. Wirel. Commun., vol. 1, no. 4, pp. 660-670, 2002.

[16] R. Saravanakumar, S. G. Susila, and J. Raja, "An energy efficient cluster based node scheduling protocol for wireless sensor networks," in SolidState and Integrated Circuit Technology (ICSICT), 2010 10th IEEE International Conference on, 2010, pp. 2053-2057.

[17] L. Qing, Q. Zhu, and M. Wang, "Design of a distributed energy-efficient clustering algorithm for heterogeneous wireless sensor networks," Comput. Commun., vol. 29, no. 12, pp. 2230-2237, 2006.

[18] T. M. Behera, S. K. Mohapatra, U. C. Samal, M. S. Khan, M. Daneshmand, and A. H. Gandomi, "Residual Energy Based Cluster-head Selection in WSNs for IoT Application," IEEE Internet Things J., vol. 6, no. 3, pp. 5132-5139, 2019.

[19] F. A. Aderohunmu, J. D. Deng, and others, "An enhanced stable election protocol (sep) for clustered heterogeneous wsn," Dep. Inf. Sci. Univ. Otago, New Zeal., 2009.

[20] D. Kumar, T. C. Aseri, and R. B. Patel, "EEHC: Energy efficient heterogeneous clustered scheme for wireless sensor networks," Comput. Commun., vol. 32, no. 4, pp. 662-667, 2009.

[21] A. Kashaf, N. Javaid, Z. A. Khan, and I. A. Khan, "TSEP: Thresholdsensitive stable election protocol for WSNs," in Frontiers of Information Technology (FIT), 2012 10th International Conference on, 2012, pp. 164-168.

[22] P. G. V. Naranjo, M. Shojafar, H. Mostafaei, Z. Pooranian, and E. Baccarelli, "P-SEP: A prolong stable election routing algorithm for energy-limited heterogeneous fog-supported wireless sensor networks," J. Supercomput., vol. 73, no. 2, pp. 733-755, 2017.

[23] T. M. Behera, U. C. Samal, and S. K. Mohapatra, "Energy Efficient Modified LEACH Protocol for IoT Application," IET Wirel. Sens. Syst., vol. 8, no. 5, pp. $223-228,2018$.

[24] M. Ye, C. Li, G. Chen, and J. Wu, "EECS: an energy efficient clustering scheme in wireless sensor networks," in Performance, Computing, and Communications Conference, 2005. IPCCC 2005. 24th IEEE International, 2005, pp. 535-540.

[25] Y. R. Tsai, "Coverage-preserving routing protocols for randomly distributed wireless sensor networks," IEEE Trans. Wirel. Commun., vol. 6, no. 4, pp. 1240-1245, Apr. 2007.

[26] D. Mahmood, N. Javaid, S. Mahmood, S. Qureshi, A. M. Memon, and T. Zaman, "MODLEACH: a variant of LEACH for WSNs," in Broadband and Wireless Computing, Communication and Applications (BWCCA), 2013 Eighth International Conference on, 2013, pp. 158163.

[27] S. Dutt, S. Agrawal, and R. Vig, "Cluster-head restricted energy efficient protocol (CREEP) for routing in heterogeneous wireless sensor networks," Wirel. Pers. Commun., vol. 100, no. 4, pp. 1477-1497, 2018.

[28] J. Wang, Y. Gao, W. Liu, A. K. Sangaiah, and H.-J. Kim, “An improved routing schema with special clustering using PSO algorithm for heterogeneous wireless sensor network," Sensors, vol. 19, no. 3, p. 671, 2019.

[29] A. P. Abidoye and I. C. Obagbuwa, "Models for integrating wireless sensor networks into the Internet of Things," IET Wirel. Sens. Syst., vol. 7, no. 3, pp. 65-72, 2017.

[30] B. Elbhiri, R. Saadane, D. Aboutajdine, and others, "Developed Distributed Energy-Efficient Clustering (DDEEC) for heterogeneous wireless sensor networks," in $I / V$ Communications and Mobile Network (ISVC), 2010 5th International Symposium on, 2010, pp. 1-4.

[31] J. Jia, Z. He, J. Kuang, and C.-X. Chen, "An energy-efficient adaptive clustering routing algorithm for wireless sensor networks," in Cross Strait Quad-Regional Radio Science and Wireless Technology Conference (CSQRWC), 2011, 2011, vol. 2, pp. 964-969.

[32] D. S. Kim and Y. J. Chung, "Self-Organization Routing Protocol Supporting Mobile Nodes for Wireless Sensor Network," in Computer and Computational Sciences, 2006. IMSCCS '06. First International Multi-Symposiums on, 2006, vol. 2, pp. 622-626.

[33] D. Kumar and R. B. Patel, "Multi-hop data communication algorithm for clustered wireless sensor networks," Int. J. Distrib. Sens. networks, vol. 7, no. 1, p. 984795, 2011.

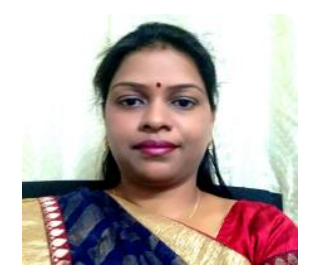

T. M. Behera got her B.Tech degree in Electronics \& Communication. Engineering from the BPUT in 2007. She received her M.Tech in Communication System from KIIT University in 2012. She has over 11 years of teaching experience and is currently an Assistant Professor in SOEE at KIIT University, Bhubaneswar. Her research area broadly includes WSNs and its application in IoT.

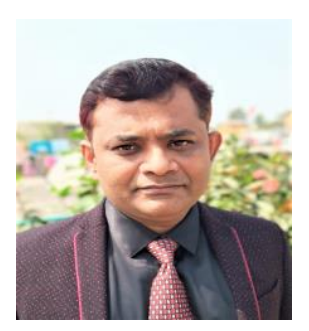

Dr. S. K. Mohapatra received his Ph.D. from NIT, Rourkela, in the year 2016. He is currently an Assistant Professor in SOEE, KIIT University, Bhubaneswar. His research interests include Modeling and Simulation of Nanoscale Devices and its application in IoT. Energy-efficient WSN, Adhoc Networks, Metamaterial absorbers in $\mathrm{THz}$ application, UWB-MIMO and Reconfigurable Antenna. He has been a part of committee member of various international conferences, Editorial Board Member and Reviewer of international journals. He is a life member of ISTE, IETE, CSI, OITS, and Senior Member of IEEE. 


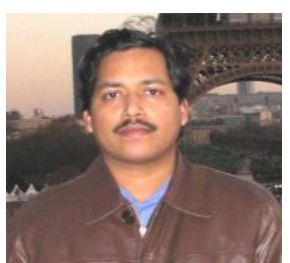

Dr. U. C. Samal received his M. Tech. in Electronic Systems and Communication Engineering from NIT, Rourkela, in 2006. He obtained his Ph.D. from the Department of Electrical Engineering, IIT, Kanpur, India in 2015. His area of interest lies in wireless communication, signal processing, and WSNs. Currently, he is working as Assistant Professor at KIIT University, Bhubaneswar, Odisha.

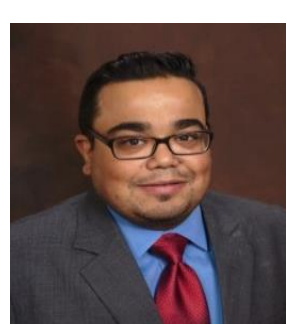

Dr. Mohammad S. Khan is currently an Assistant Professor of Computing at East Tennessee State University and the director of Network Science and Analysis Lab (NSAL). He received his M.Sc. and Ph.D. in Computer Science and Computer Engineering from the University of Louisville, Kentucky, USA, in 2011 and 2013, respectively. His primary area of research is in ad-hoc networks, network tomography, connected vehicles, and vehicular social networks. He currently serves as Co-Editor-in-Chief of International Journal of Grid and High-Performance Computing (IJGHPC) and as an associate editor of IEEE Access. He has been on technical program committees of various international conferences and technical reviewer of various international journals in his field. He is a member of IEEE.

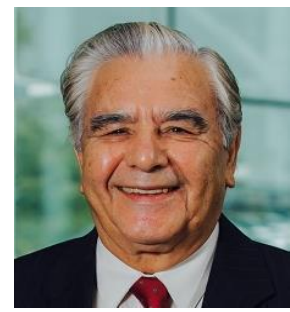

Dr. Mahmoud Daneshmand is Co-Founder and Professor of Department of Business Intelligence \& Analytics; and Professor of Department of Computer Science at Stevens Institute of Technology. He has more than 40 years of Industry \& University experience as Professor, Researcher, Assistant Chief Scientist, Executive Director, Distinguished Member of Technical Staff, Technology Leader, Chairman of Department, and Dean of School at: Bell Laboratories; AT\&T Shannon Labs-Research; University of California, Berkeley; University of Texas, Austin; Sharif University of Technology; University of Tehran; New York University; and Stevens Institute of Technology. He received his Ph.D. and M.S. degrees in Statistics from the University of California, Berkeley; M.S. and B.S. degrees in Mathematics from the University of Tehran.

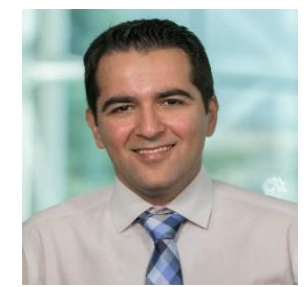

Dr. Amir H. Gandomi is an Assistant Professor of Analytics \& Information Systems at School of Business, Stevens Institute of Technology. Prior to joining Stevens, Dr. Gandomi was a distinguished research fellow in headquarter of BEACON NSF center located at Michigan State University. He received his Ph.D. in engineering and used to be a lecturer in several universities. Dr. Gandomi has published over one hundred and thirty journal papers and four books. Some of those publications are now among the hottest papers in the field and collectively have been cited more than 11,000 times ( $\mathrm{h}$-index $=52$ ). He has been named as Highly Cited Researcher (top 1\%) for two consecutive years, 2017 and 2018, and one of the world's most influential scientific minds. Dr. Gandomi is currently ranked 20th in GP bibliography among more than 11,000 researchers. He has also served as associate editor, editor and guest editor in several prestigious journals and has delivered several keynote/invited talks. Dr. Gandomi is part of a NASA technology cluster on Big Data, Artificial Intelligence, and Machine Learning. His research interests are global optimization and (big) data mining using machine learning and evolutionary computations in particular. 